\title{
LITHIUM-6 NUCLEOSYNTHESIS IN THE ISM
}

\author{
ROBERT A. MALANEY \\ Canadian Institute for Theoretical Astrophysics \\ University of Toronto, Toronto, ON, CANADA M5S $1 A 7$.
}

The nucleosynthesis of ${ }^{6} \mathrm{Li}$ in the galaxy has become of considerable interest in the past year. This is largely because of the exciting developments relating to the first detection of this isotope outside of our own solar system. Not only has ${ }^{6} \mathrm{Li}$ been recently detected in the atmospheres of halo dwarfs [1-2], but also in the local interstellar medium (ISM) [3-4].

It is well known that knowledge of the primordial abundance of the lithium isotopes has important ramifications for cosmology, through the constraints they impose on standard big bang nucleosynthesis (SBBN) [5], and those imposed on non-standard primordial nucleosynthesis models. Since a complete understanding of the galactic evolution of the lithium isotopes is a prerequisite to unambiguously determining their primordial abundance, a thorough interpretation of the new ${ }^{6} \mathrm{Li}$ data in the context of chemical evolution models cannot be overstated.

Here we wish to focus our attention on the recent ISM observations of the ${ }^{7} \mathrm{Li} /{ }^{6} \mathrm{Li}$ ratio. In ref. $[3]{ }^{7} \mathrm{Li} /{ }^{6} \mathrm{Li}=12.5_{-3.4}^{+4.3}$ is reported toward $\rho \mathrm{Oph}$, whereas in ref. [4] ${ }^{7} \mathrm{Li} /{ }^{6} \mathrm{Li}=6.8_{-1.7}^{+1.4}$ is reported toward $\zeta \mathrm{Oph}$ and ${ }^{7} \mathrm{Li} /{ }^{6} \mathrm{Li}=5.5_{-1.1}^{+1.3}$ is reported toward $\zeta$ Per. As pointed out by these two groups, the inferred ${ }^{7} \mathrm{Li} /{ }^{6} \mathrm{Li}$ ratios seem at odds with current chemical evolution models. Such models predict that since the epoch of solar system formation $4.6 \mathrm{Gyr}$ ago, the ${ }^{7} \mathrm{Li} /{ }^{6} \mathrm{Li}$ ratio should have increased significantly in value from the measured solar system value of $12.6 \pm 0.2$. The principal reason for the predicted rise in the ${ }^{7} \mathrm{Li} /{ }^{6} \mathrm{Li}$ ratio over the past $4.6 \mathrm{Gyr}$ is the widely advocated (but not yet unambiguously identified) stellar source of ${ }^{7} \mathrm{Li}$ - postulated to account for the order of magnitude difference in the Pop II and Pop I lithium abundances. That the ISM ${ }^{7} \mathrm{Li} /{ }^{6} \mathrm{Li}$ ratio has not increased significantly, and in fact seems to have decreased over the past 4.6 Gyr (if the observations of [4] are taken at face value), presents a serious conundrum. 
We outline a remedy to this potential conflict between observation and theory. We suggest that if a stellar source of lithium which is rich in both ${ }^{7} \mathrm{Li}$ and ${ }^{6} \mathrm{Li}$ is invoked, then chemical evolution models which predict a flat or decreasing ${ }^{7} \mathrm{Li} /{ }^{6} \mathrm{Li}$ ratio over the past $4.6 \mathrm{Gyr}$ can be constructed. We discuss the feasibility of this hypothesis, and ask whether the energetics of such a process can be consistent with some type of flare activity in the vicinity of a stellar source.

The total energy, $E_{T}$, required for stellar ${ }^{6} \mathrm{Li}$ production can be written

$$
E_{T} \sim f \frac{M}{10 M_{\odot}} \frac{\eta}{\mathrm{ergs}} 10^{58} \quad \text { ergs },
$$

where $\eta \sim 1 \mathrm{erg}$ is the energy needed to produce one lithium atom, $f$ is the fraction of the observed ${ }^{6} \mathrm{Li}$ in the ISM ascribed to the stellar source, and $M$ is the total mass of ${ }^{6} \mathrm{Li}$ in the present ISM. A value of $M \sim 20 M_{\odot}$ would correspond to the abundance of ${ }^{6} \mathrm{Li} / \mathrm{H} \sim 3 \times 10^{-10}$ (inferred by [3]) being representative of the entire ISM, and not just some local abundance of the particular ISM clouds observed. We caution, however, that the determination of absolute ISM lithium abundances is subject to large ionization corrections and grain-depletion factors, which do not influence the determination of the isotopic ratio in any significant way. With these uncertainties in mind, we note that $M \sim 20 M_{\odot}$ is quite uncertain; although twice this amount is likely to be a reasonable (but not firm) upper limit. We see then that $E_{T}$ can be as low as $10^{57} \mathrm{ergs}$, even for the case $f=1, M \sim 20 M_{\odot}$. Although $10^{47} \mathrm{ergs} /$ year is a large amount of energy, it is still below the energy that can be input to the ISM from different sources in the galaxy (eg. flare stars, supernovae), and suggests that the hypothesis of a stellar source of ${ }^{6} \mathrm{Li}$ observable in the present ISM is certainly possible. Again, however, it is clear that an unambiguous identification of the stellar ${ }^{6} \mathrm{Li}$ source is necessary in order to properly calculate the energetic requirements.

\section{References}

[1] Smith, V. V., Lambert., D. L., and Nissen, P. E., 1993, ApJ., 408, 262.

[2] Hobbs, L. M. and Thorburn, J. A., 1994, ApJL, 428, L25.

[3] Lemoine, M., Ferlet, R., Vidal-Madjar, A., Emerich, C. and Bertin, P., 1993, Astron. Astrophys. 269, 469.

[4] Meyer, D., Hawkins, I. and Wright, E. L., 1993, ApJL, 409, L61.

[5] A complete list of references for this paper can be found in the full length version :Malaney, R. A., 1994, Memorie Soc. Astr. It., Ed. R. Pallavicini (CITA/94/29). 